\title{
Why is the Acupuncture Point Bai Huei (Gv20) Point of Union?
}

\author{
Dr. Maria Kuman* \\ Holistic Research Institute, USA \\ *Corresponding author: Maria Kuman PhD, Holistic Research Institute, 1414 Barcelona, Knoxville, TN 37923, USA; Email holisticare1@gmail.com
}

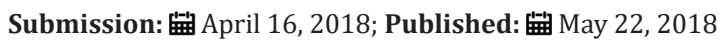

\begin{abstract}
Ancient Chinese texts claim that the point Bai Huei on top of the head is a point of union: 101 imbalances can make it painful and 101 diseases can be healed by treating it with acupuncture. This article aims to explain why this is so. Our nonlinear electromagnetic field (NEMF) has a donut (torus) shape. Recently, the Russians patented equipment capable to measure its spinning. The axis of spinning is passing through the hole of the donut, which is along the backbone. This makes our body a magnet with northern pole on top of the head and southern pole at the tailbone. Our donut-shaped NEMF has 6 spinning energy centers along the axis of spinning, which rule the 6 endocrinal glands; the seventh one is Bai Huei. Each of these spinning centers, when numbered from the tailbone to the head, is represented by a donut with increasing radius. All these donuts have the same axis of spinning, which makes them united on top of the head and at the tailbone. In vertical cross-section, they look like 6 magnetic lines starting at the top of the head and finishing at the tailbone (Figure 1). This makes the top of the head, where the northern pole of the magnet is, a point of union.
\end{abstract}

Keywords: Acupuncture; Acupuncture point Bai Huei; Bai Huei a point of union; Explaining the point of union; Shape of human nonlinear EMF

\section{Introduction}

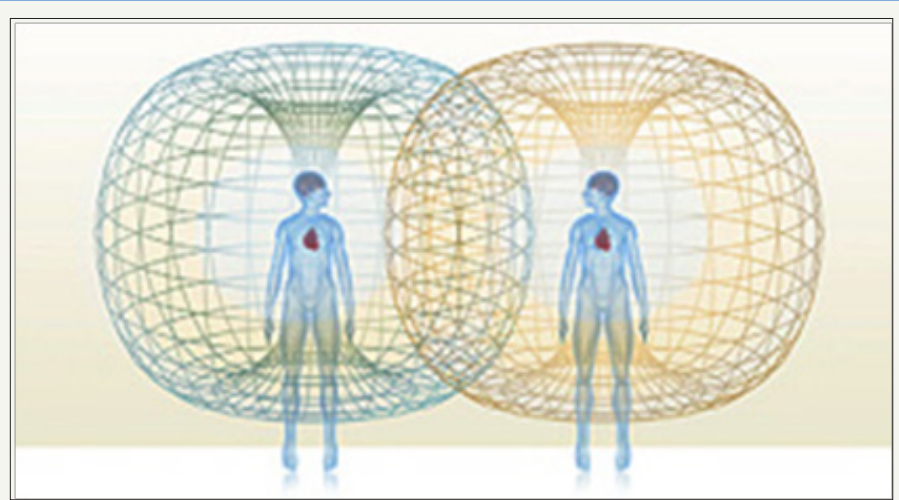

Figure 1: The nonlinear EMF of the heart as measured by the heart math institute.

Our nonlinear EMF has the shape of a donut (torus), just like the EMF of our Sun and Earth. In the US, the heart math institute in California first measured the shape of the heart's EMF and found that it has a donut shape (Figure 1) [1].

Let us introduce some concepts of nonlinear physics, which we would need. If the bottom of a river has stones, the flux of running water would be nonlinear because it needs to flow around the stones. Behind the stones, turbulence would be observed manifested with a couple of: vortex spinning clockwise and antivortex spinning counterclockwise.

Following the law of the folded fingers of the right hand in physics, when the folded fingers show the direction of the current, the thumb show the direction of the induced magnetic field. Following this law, the vortices (which spin clockwise) would induce magnetic field toward the surface. This would make the vortices to suck energy in. Following the same law, the anti-vortices (which spin counterclockwise) would induce magnetic field off the surface, which would make the anti-vortices to emit energy.

\section{The dynamic of our spinning nonlinear EMF revealed} from russian and author's measurements

The Russian scientist Shkatov [2] first measured the spinning of the human donut-shaped EMF with his patented equipment called 'torsemeter.' He found that positive emotions make the donut spin faster clockwise, while negative emotions make the donut spin faster counterclockwise. 
Measurements of the author [3] with her patented sensitive energy meter found that positive emotions (or just positive thinking) increase the body energy and improve the energy balance, which means positive emotions make us healthier (Figure
2). Measurements found that negative emotions (or just negative thinking) decrease the body energy and worsen the energy balance (Figure 2).
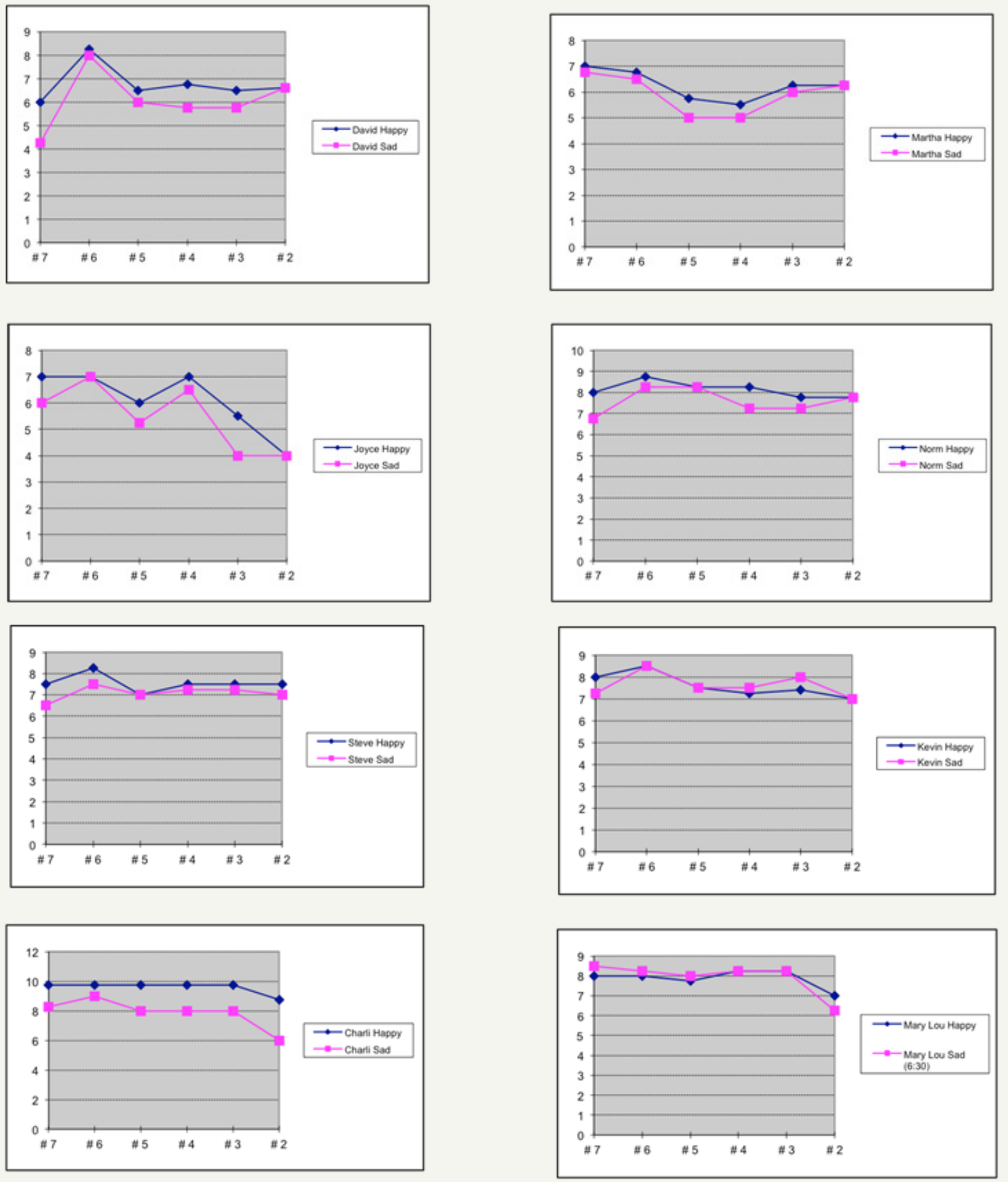

Figure 2: The measurements with our patented sensitive energy meter showed that:

i. Positive emotions (or just positive thinking) increase the human energy and improve the energy balance, which makes the positive thinking person healthier.

ii. Negative emotions (or just negative thinking) decrease the human energy and worsen the energy balance, which is a step toward a disease. 
We found that at negative thinking the genetically inherited weak organ drops in energy maximum. This means that negative emotions (or just negative thinking) take us a step farther to a chronic disease of the genetically inherited weak organ. (The measured points on the horizontal axis of Figure 2 are shown on Figure 3). This is in full agreement with the finding of Selye [4] that the same stress causes chronic disease of different organs in different individuals and that the genetically inherited weak organ collapses under stress first, just like in engineering stress would crack the material at the weakest place.

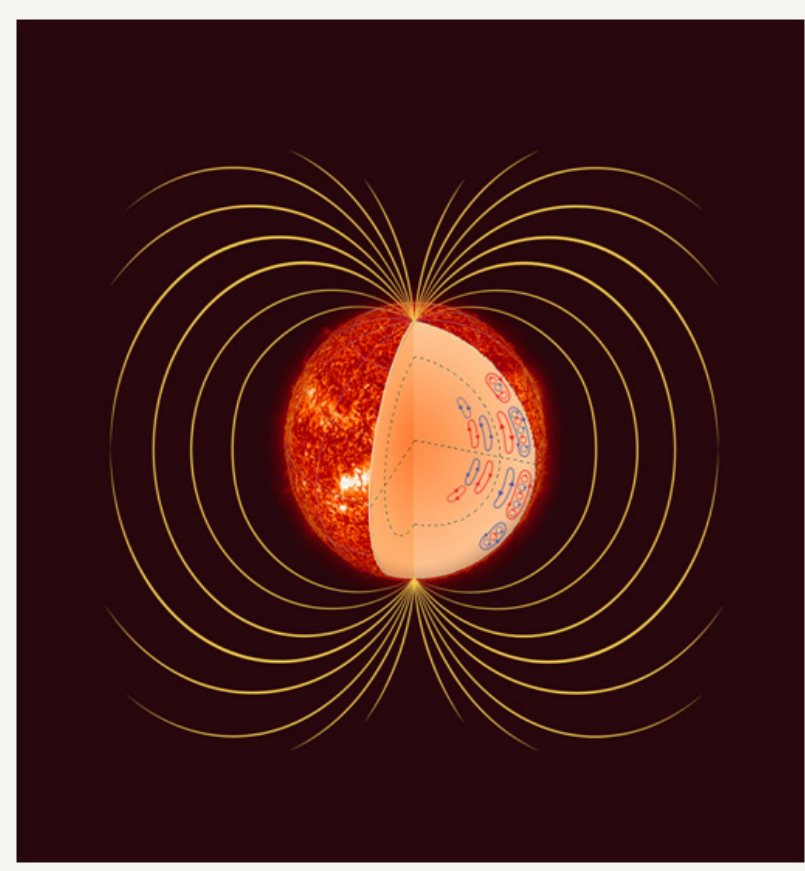

Figure 3: Sun's torus-shaped nonlinear EMF (vertical cross-section). Visible are the ring currents induced by the sun's spinning, which crank the magnetic field.

In full agreement with the findings of Selye [4], we found with our measurements that: at negative (sad) thinking the heart dropped in energy maximum for David and Norm and they both said that their fathers died of heart attack; at negative (sad) thinking the thyroid gland of Martha dropped in energy maximum and she told us that her mother had thyroid problem; when we told Joyce that we found with our measurements that her stomach is most sensitive to stress, she said she knows that - each time she gets upset, her stomach gets upset.

\section{Quantum energy levels and torus-shaped EMF-How common are they?}

Not only are the nonlinear EMF of humans, Sun, and Earth torus-shaped, the EMF of helium nanodroplets [5,6] and even elementary particles seems to have the same donut shape EMF with the same dynamic. The elementary particles emit from their equatorial area virtual photons and swallow them back, just like our Sun emits spinning energy balls from its anti-vortices, which after a loop trajectory are swallowed back by adjacent vortices [7] (Figure 4).

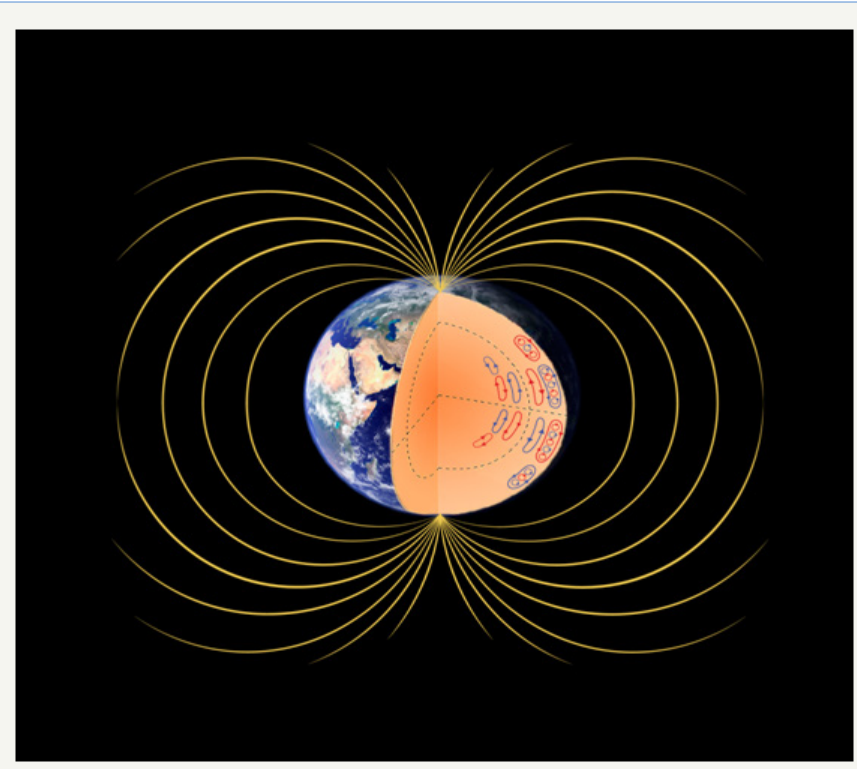

Figure 4: Earth's torus-shaped nonlinear EMF (vertical cross-section). Visible are the ring currents induced by the earth's spinning, which crank the magnetic field.

Not only are the energy levels of elementary particles, atoms, and molecules discrete or quantized, looking at the radiation belts of our Earth we see discrete levels and looking at our Sun we see discrete orbits. Even the rings of Saturn are discrete. The same is the situation with the human EMF-our energy levels are discrete (Figure 5) (See also the book of M Kuman, Quantum Mind and Quantum Growth) [7].

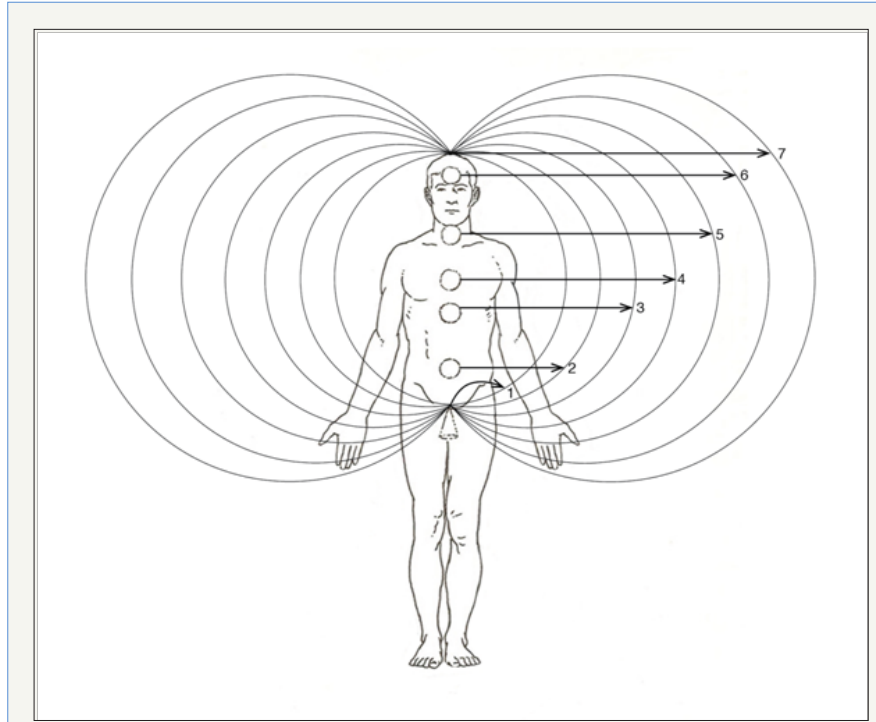

Figure 5: The human donut-shaped nonlinear EMF in vertical cross-section. The point Bai Huei on top of the head integrates (sums up) the energies of the underlying 6 alternating vortices and anti-vortices. Their discrete basic quantum energy levels sum up at the point Bai Huei.

The dynamic of helium nanodroplets seems to be alternating shifts between two shapes: i) the shape of torus when the nanodroplets spin fast around axis passing through the hole of 
the donut, are bulged at the equator, and exhibit turbulence with observed Bragg's neutron scattering; ii) a state of low equatorial activity, when Bragg's neutron scattering is not observed. X-rays diffraction revealed that the nanodroplets are shrunk at the equator; they are elongated and emit energy from both ends of the axis of spinning [7].

What caused the shift to the second state? It seems that as the helium droplet was spinning faster and faster, the bulging at the equator reached the critical value of pressure, which the helium substance can tolerate. To release the pressure, the droplets started spinning in opposite direction, the magnetic poles of the donut distanced each other, energy was emitted through the poles (observed with X-ray diffraction), and the turbulent activity in the equatorial area seized (Bragg's neutron scattering was not observed).

Non-linear physics need to step in to finish the picture of the dynamic. During the active cycle, the helium nanodroplets are torus-shaped, spin faster and faster, suck energy through the poles, bulge at the equator, and exhibit turbulence there. For this to happen, the donut needs to spin clockwise like a vortex. Then both magnetic poles (of the induced by the spinning EMF) would be vortices sucking energy in [7].

When the bulging at the equator reaches the critical value of pressure, which the substance of the helium nanodroplets can tolerate, to release the pressure the spinning must switch from clockwise to counterclockwise. Then the two magnetic poles would be anti-vortices emitting energy. Indeed, the X-rays diffraction from the helium nanodroplets revealed emission of energy, which ended the turbulent activity.
This dynamic seems to be typical for all torus-shaped electromagnetic fields (EMF) including our Sun. The alternative switches between high solar activity (warm periods on Earth) and low solar activity (Ice Ages on Earth) must be a product of these switches from clockwise spinning of the Sun during the warm periods to counterclockwise spinning during the Ice Ages.

The astronomers speak about this as periodic reversal of the magnetic polarity of the sun. However, this actually means periodic reversal of the direction of spinning of the sun. When the sun spin clockwise, it sucks energy in, spins faster and faster, becomes bulged at the equator, and exhibit turbulence in the equatorial area seen as solar spots. This is the period of high solar activity [8].

When the bulging at the equator reaches the critical value of pressure, which the solar plasma can tolerate, to release the pressure the sun stars spinning in opposite (counterclockwise) direction. The two magnetic poles start emitting energy and distance each other. The sun shrinks at the equator and its turbulent activity ends. This continues until the pressure at the equator reaches a critical value. To release the pressure, the sun will start spinning in opposite (clockwise) direction and suck energy, and this goes on and on.

In the human body, emotions rule the direction of spinning. Positive emotions make the human donut-shaped EMF spin clockwise, at which energy is sucked through the poles of the donut, which makes us feel uplifted. Negative emotions make the donut spin counterclockwise, at which energy is released through the poles of the donut. If so, to lift up our energy level, we must do our best to experience only positive emotions.

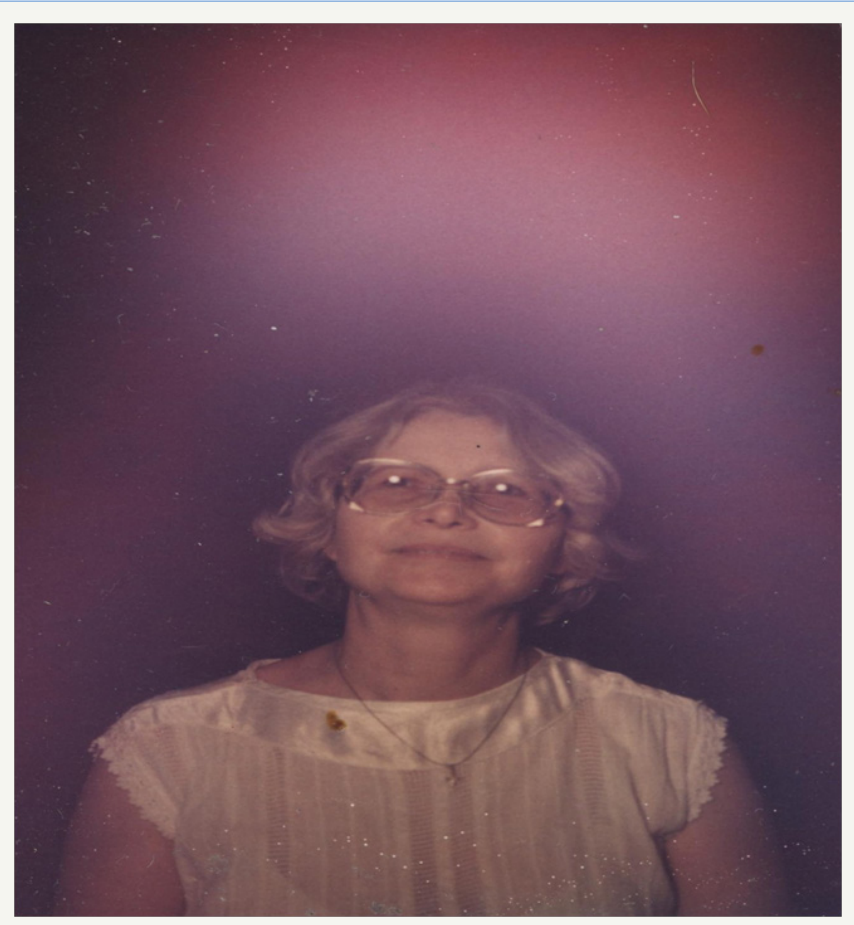

Figure 6: Kirlian photo of the author's nonlinear EMF taken in 1991. High frequency electric field was used to multiply the photons and make the photographing of the EMF possible. 
Figure 6 pictures the active energy level 9 [7]. Beside the basic seven levels depicted on Figure 5 possible are 5 more discrete quantum levels $8,9,10,11$, and 12 . They are light ball over the top of the head at a growing height.

\section{Conclusion}

Recently, the Russians patented sensitive equipment, which they called 'torsemeter', capable to measure the spinning of the human donut-shaped EMF. The axis of spinning is passing through the hole of the donut, which is along the backbone. This makes our body a magnet with northern pole on top of the head and southern pole at the tailbone. Our donut-shaped nonlinear EMF has 7 spinning energy centers (alternating vortex-anti-vortex-vortex, etc.) along the axis of spinning.

The seventh one on top of the head is Bai Huei, which integrate the energies of the lying under it 6 spinning energy centers, which rule and regulate the 6 endocrinal glands of the body. Each of these spinning centers, when numbered from the tail to the head, is represented by a donut with increasing radius. All these donuts have the same axis of spinning, which makes them united on top of the head and at the tailbone. In vertical cross-section, they look like 6 magnetic lines starting at the top of the head and finishing at the tailbone (Figure 5). This makes the top of the head, where the northern pole of the magnet is, a point of union.

\section{References}

1. www.HeartMath.com

2. Shkatov, Tihoplav V, Miroznanie (2012) Saint Petersburg, Russia.

3. Kuman M (2005) Science speaks of god. Health and Happiness Books.

4. Selye H (1976) Stress in health and disease. Nutterworth, BostonLondon, UK.

5. Smart A (2014) Physics today. 67(11): 16.

6. Baron J (2014) Science. 343:269.

7. Kuman M (2015) Quantum Mind and Quantum Growth. Health and Happiness Books.

8. Kuman M (2017) Glimpse to future physics and cosmology. Health and Happiness Books.
Creative Commons Attribution 4.0 International License

For possible submissions Click Here

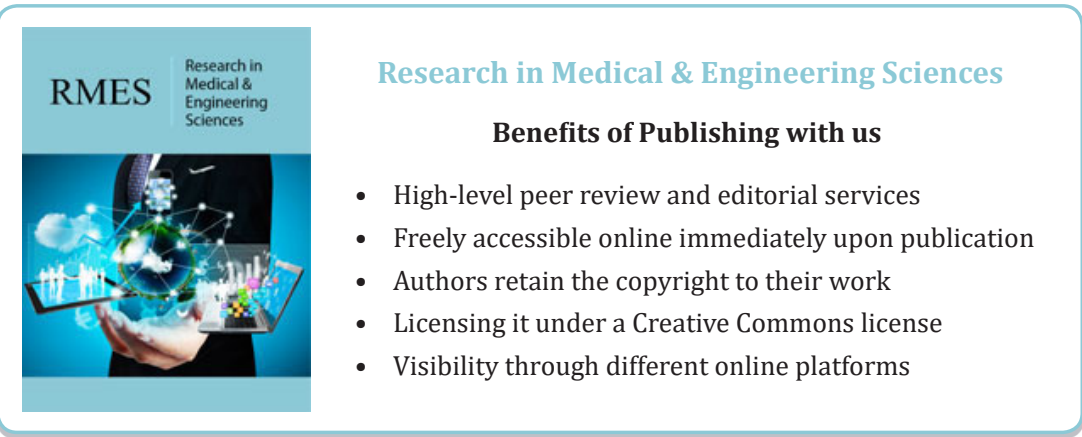

